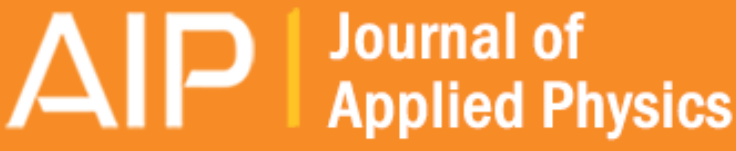

\section{Breakdown of intermediate range order in AsSe chalcogenide glass}

Azkar S. Ahmad, K. Glazyrin, H. P. Liermann, H. Franz, X. D. Wang, Q. P. Cao, D. X. Zhang, and J. Z. Jiang

Citation: Journal of Applied Physics 120, 145901 (2016); doi: 10.1063/1.4964798

View online: http://dx.doi.org/10.1063/1.4964798

View Table of Contents: http://scitation.aip.org/content/aip/journal/jap/120/14?ver=pdfcov

Published by the AIP Publishing

\section{Articles you may be interested in}

Structural, dynamic, electronic, and vibrational properties of flexible, intermediate, and stressed rigid As-Se glasses and liquids from first principles molecular dynamics

J. Chem. Phys. 141, 194506 (2014); 10.1063/1.4901515

Short and intermediate range order of Ge 20 Se $80-x$ Te $x$ glasses

J. Appl. Phys. 108, 073505 (2010); 10.1063/1.3488907

AsS: Bulk inorganic molecular-based chalcogenide glass

Appl. Phys. Lett. 91, 031912 (2007); 10.1063/1.2759261

Studies on structural, electrical, and optical properties of $\mathrm{Cu}$ doped As-Se-Te chalcogenide glasses

J. Appl. Phys. 101, 063520 (2007); 10.1063/1.2712162

Structural properties of the glass system As-Se-S studied by x-ray absorption spectroscopy

J. Appl. Phys. 88, 2533 (2000); 10.1063/1.1286109

Pure Metals • Ceramics Alloys - Polymers in dozens of forms
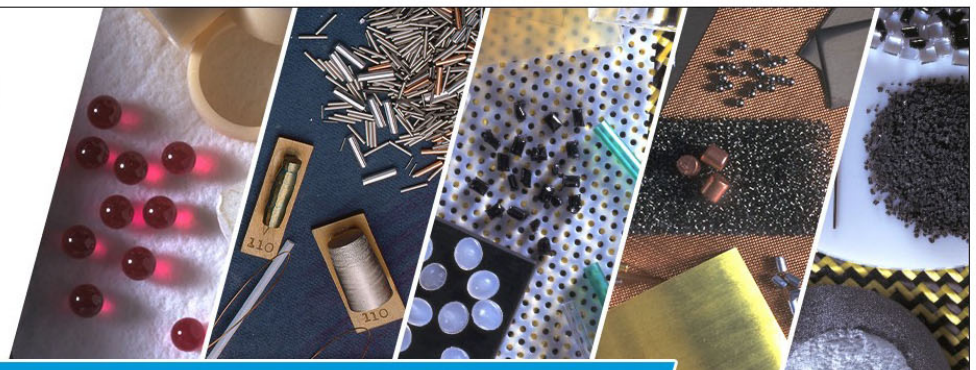


\title{
Breakdown of intermediate range order in AsSe chalcogenide glass
}

\author{
Azkar S. Ahmad, ${ }^{1}$ K. Glazyrin, ${ }^{2}$ H. P. Liermann, ${ }^{2}$ H. Franz, ${ }^{2}$ X. D. Wang, ${ }^{1}$ Q. P. Cao, ${ }^{1}$ \\ D. X. Zhang, ${ }^{3}$ and J. Z. Jiang ${ }^{1, a)}$ \\ ${ }^{1}$ International Center for New-Structured Materials and Laboratory of New-Structured Materials, State Key \\ Laboratory of Silicon Materials, School of Materials Science and Engineering, Zhejiang University, \\ Hangzhou 310027, People's Republic of China \\ ${ }^{2}$ Photon Science, Deutsches Elektronen-Synchrotron DESY, Notkestraße 85, D-22603 Hamburg, Germany \\ ${ }^{3}$ State Key Laboratory of Modern Optical Instrumentation, Zhejiang University, Hangzhou 310027, \\ People's Republic of China
}

(Received 24 June 2016; accepted 1 October 2016; published online 13 October 2016)

\begin{abstract}
As-cast amorphous AsSe (a-AsSe) has been characterized by in-situ high pressure XRD and Raman spectroscopy up to the pressure of $\sim 30 \mathrm{GPa}$ using diamond anvil cell together with ab-initio molecular dynamics simulations. A gradual densification has been observed under compression along with the breakdown of intermediate range ordering at $\sim 16 \mathrm{GPa}$. The whole transformation process can be divided into three relatively distinct pressure regimes from 1 bar to $7 \mathrm{GPa}$, from 7 to $16 \mathrm{GPa}$, and beyond $16 \mathrm{GPa}$. Our XRD results together with Raman spectroscopic studies confirm that in the a-AsSe pressure tuning results in network transformations only, without sudden jump in the density. The results obtained by high pressure ab-initio molecular dynamics simulations demonstrate the variations in the local structures associated with the experimentally observed transformations. The amorphous-to-amorphous network transformation is found to be reversible upon decompression. Published by AIP Publishing. [http://dx.doi.org/10.1063/1.4964798]
\end{abstract}

\section{INTRODUCTION}

Pressure can tune the structure and hence the properties of a material. Many pressure-induced transitions such as amorphous-to-crystalline, ${ }^{1-4}$ crystalline-to-amorphous, ${ }^{5-7}$ amorphous-to-amorphous, ${ }^{8,9}$ liquid-to-liquid, ${ }^{10}$ metal-tosemiconductor, ${ }^{11}$ and metal-to-insulator ${ }^{12}$ transitions have been widely reported in a variety of materials. The behavior of amorphous materials under pressure is complex because of their metastability as compared to their thermodynamically stable crystalline counterparts. ${ }^{13}$ In amorphous materials, pressure-induced amorphous-to-amorphous transitions have attracted a great deal of attention of high pressure, condensed matter physics, and materials scientists. Many unusual amorphous-to-amorphous transitions have been reported in a number of network amorphous materials and metallic glasses. ${ }^{14-17}$ Among all amorphous materials, amorphous chalcogenides exhibit amorphous-to-amorphous network transitions under pressure, as they undergo compression because of their relatively open structure at ambient condition as compared to the metallic glasses which are closely packed. High pressure studies on liquid and glassy $\mathrm{Ge}_{\mathrm{x}} \mathrm{Se}_{1-\mathrm{x}}$ and $\mathrm{Ge}_{\mathrm{x}} \mathrm{S}_{1-\mathrm{x}}$ are the best examples of such network transitions in liquid and glassy chalcogenides. Vaccari et al. reported hysteresis in reversible amorphous-to-amorphous transition in $\mathrm{GeS}_{2}$ using X-ray absorption fine structure (XAFS) and X-ray diffraction (XRD) measurements. ${ }^{18}$ Theoretical study on a-GeSe $\mathrm{Se}_{2}$ has shown a gradual pressure-induced insulatorto-metal transition and a gradual amorphous-to-amorphous transition from fourfold-to-sixfold Ge coordination and from twofold-to-fourfold Se coordination. ${ }^{19}$ In another study, ${ }^{20}$

\footnotetext{
a) Author to whom correspondence should be addressed. Electronic mail: jiangjz@zju.edu.cn
}

$\mathrm{GeSe}_{2}$ glass was observed to undergo densification upon pressurization up to $\sim 3 \mathrm{GPa}$ through conversion of edge- to corner-sharing tetrahedra, and at pressures $\geq 4 \mathrm{GPa}$ the densification was observed that was contributed to the increase of the coordination numbers around Ge and Se atoms. By performing in situ high pressure XRD measurements on $\mathrm{GeSe}_{4}$, Skinner et al. found the destruction of intermediate range order (IRO) under compression. ${ }^{21}$ Recently, Kalkan et al. reported polyamorphism and metallization in tetrahedral $\mathrm{GeSe}_{4}$ glass. $^{22}$

Apart from the conventional $\mathrm{Ge}_{\mathrm{x}} \mathrm{Se}_{1-\mathrm{x}}$ and $\mathrm{Ge}_{\mathrm{x}} \mathrm{S}_{1-\mathrm{x}}$ glassy systems, As-S and As-Se glassy systems are well known glass formers. Among all As-Se and As-S glassy systems, isostructural $\mathrm{As}_{2} \mathrm{Se}_{3}$ and $\mathrm{As}_{2} \mathrm{~S}_{3}$ are relatively well studied at ambient conditions. Their glassy structure is characterized by a prepeak of the first sharp diffraction peak (FSDP) in X-ray diffraction (XRD) patterns, which is indicative to the intermediate range order. ${ }^{23-27}$ In glassy and liquid $\mathrm{As}_{2} \mathrm{~S}_{3}$, the prepeak disappeared as pressure increased, hereby confirming the destruction of intermediate range ordering. ${ }^{28,29}$ However, in these transitions, no sudden drop in the density has been found, and hence the transition was observed to be purely related to a change in the network (i.e., from a loosely packed structure to more packed one). Very recently, in Ge doped As-S glassy system (i.e., in $\mathrm{Ge}_{2.5} \mathrm{As}_{51.25} \mathrm{~S}_{46.25}$ glass), ${ }^{30}$ Kalkan et al. reported a reversible amorphous-to-amorphous transition from a molecular network to a more closely packed network. In contrast to As-S glassy systems, As-Se glassy systems have been poorly studied at high pressure. To date, $\mathrm{g}-\mathrm{As}_{2} \mathrm{Se}_{3}$ is the only candidate among all the As-Se glassy systems that has been studied under high pressure conditions. But these studies were limited to the resistivity measurements ${ }^{31}$ and band gap measurements. ${ }^{32}$ While all other As-Se 
glassy systems remain unexplored under high pressure conditions. Here, we report the results of in-situ high pressure synchrotron XRD, Raman spectroscopy measurements, and $A b$ initio molecular dynamics (AIMD) simulations on stoichiometric amorphous AsSe (a-AsSe). We observed a reversible destruction of intermediate range ordering, and hence the conversion of a quasi-layered (2D) to a closely packed (3D) network amorphous.

\section{EXPERIMENTAL AND COMPUTATIONAL METHODS}

Sample preparation: The residual oxygen content in arsenic and selenium samples of $99.999 \%$ purity was reduced using the volatilization technique. The vapor pressure of the oxides of these metals is greater than that of the metals; this has been exploited to remove the oxide species. Selenium was heated at $250{ }^{\circ} \mathrm{C}$ and arsenic at $290^{\circ} \mathrm{C}$ under vacuum for several hours. After this treatment, the required amounts of As and Se were sealed in a silica tube under vacuum. This mixture was further heated at $650{ }^{\circ} \mathrm{C}$ for $12 \mathrm{~h}$ in a rocking furnace to ensure good mixing and homogenization of the liquids. To condense a maximum of vapor into the liquid, the temperature was reduced to $500^{\circ} \mathrm{C}$ for $1 \mathrm{~h}$. Then, the ampoules were quenched in air and later annealed near the glass-transition temperature $\left(T_{\mathrm{g}} \approx 175^{\circ} \mathrm{C}\right)$ of $\mathrm{As}_{50} \mathrm{Se}_{50}$ (AsSe) glass for $10 \mathrm{~h}$ to reduce the mechanical stresses induced during the cooling. The amorphous nature of the AsSe sample was confirmed by X-ray diffraction and differential scanning calorimetry.

In-situ measurements at high pressure: Synchrotron radiation XRD and Raman spectroscopic measurements were performed in a Mao-Bell type diamond anvil cell (DAC) with a culet $300 \mu \mathrm{m}$ in diameter. The sample chamber was a hole of $\sim 120 \mu \mathrm{m}$ diameter drilled in a pre-indented T301 stainless steel gasket. The specimen was loaded into the sample chamber along with ruby as a standard for pressure calibration. A 4:1 mixture of methanol:ethanol was used as a pressure-transmitting media for both the in-situ high pressure XRD and Raman spectroscopic measurements.

Angle-dispersive XRD measurements were performed in-situ under high pressure at the Extreme Conditions Beamline (ECB) P02.2, PETRAIII, DESY, Hamburg, Germany (Liermann et al., 2015). The energy of the synchrotron radiation was adjusted to $42.7 \mathrm{keV}$. Two-dimensional diffraction patterns were collected using a Perkin Elmer XRD 1621 ScI-bonded amorphous silicon 2D detector $(2048 \times$ 2048 pixels, $200 \times 200 \mu$ m pixel size) mounted orthogonal to the direction of the incident $\mathrm{X}$-ray beam. $\mathrm{CeO}_{2}$ standard (NIST 674 b) was used to measure the sample-to-detector distance and tilt of the detector relative to the beam path. The sample was exposed to an X-ray beam with a diameter of $8(\mathrm{H}) \times 3(\mathrm{~V}) \mu \mathrm{m}^{2}$ for $1 \mathrm{~min}$. The background was collected by exposing X-ray beam on the diamonds of the empty cell (the same cell which was used for the in-situ high pressure XRD measurements) which was later used for background subtraction. This method of collecting background has been successfully applied to various other studies and is found to be efficient and accurate. The two-dimensional patterns were integrated into $Q$-space using the software package Fit2D. ${ }^{33}$
Raman experiments were performed using a Renishaw microscope employing a laser of wavelength $785 \mathrm{~nm}$. The laser power was set to $5 \mathrm{~mW}$ after optimization to avoid heat- and photo-induced degradation, while still enabling the collection of high-quality Raman spectra.

Ab-initio molecular dynamics simulations: Ab-initio molecular dynamics (AIMD) simulations were performed using the Vienna ab-initio simulations package (VASP) $\operatorname{code}^{34}$ and the projected augmented wave method (PAW) ${ }^{35,36}$ with generalized gradient approximations. A supercell containing 192 atoms (96 As and $96 \mathrm{Se}$ ) was quenched from $2000 \mathrm{~K}$ to room temperature $(\mathrm{RT})$ at $0.33 \mathrm{~K} / \mathrm{fs}$, and the Nose thermostat $^{37}$ was used to control the temperature. The mass density was fixed at the measured value for a-AsSe, and the zero-pressure density was obtained by relaxing the box. After quenching to RT, the system was equilibrated for 4000 time steps ( $3 \mathrm{fs} / \mathrm{step}$ ), and pressure was applied by uniformly reducing the volume of the box. This method of application of pressure mimics experimental hydrostatic compression and has been previously adopted in many AIMD studies of pressureinduced structural changes. When comparing simulations with experimental results, the spatio-temporal limitation of the AIMD simulations must be taken into account. In the present studies, the small size of the box (192 atoms) may cause statistical fluctuations and lead to different structures. However, the general trend in structural evolution can still be identified.

\section{RESULTS AND DISCUSSION}

Figure 1 shows XRD patterns of a-AsSe during compression. Under ambient conditions and moderate pressures, there is a pre-peak at $2 \theta \approx 3.2^{\circ}$ in the XRD patterns; this corresponds to intermediate range ordering, i.e., quasi-layered (2D) structure. As the pressure is increased more significantly, the pre-peak shifts to higher $2 \theta$ values and loses intensity, finally disappearing at $\sim 16.6 \mathrm{GPa}$ (Fig. 1). The loss of this peak reflects the destruction of the intermediate range order (IRO) associated with the quasi-layered (2D) structure and marks the completion of the transformation

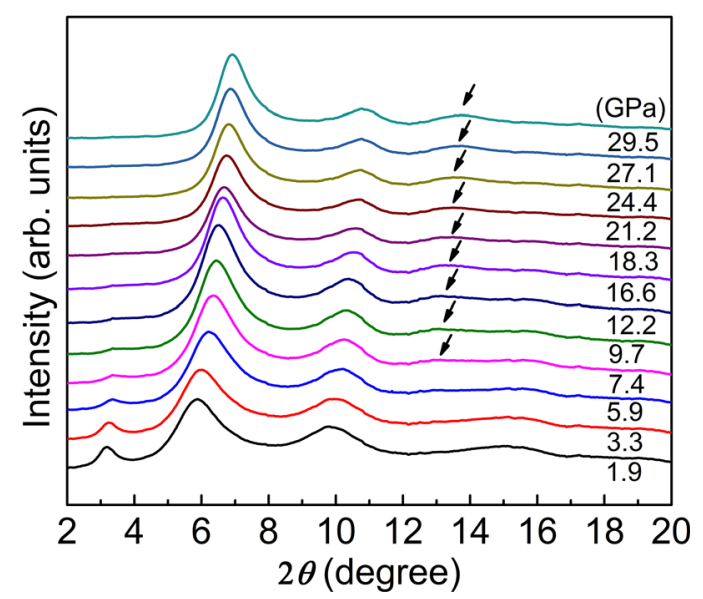

FIG. 1. In-situ high-pressure XRD patterns of a-AsSe at room temperature in a diamond-anvil cell. Integrated XRD patterns at selected pressures during compression up to $29.5 \mathrm{GPa}$. The black arrows indicate the appearance of a new feature in XRD patterns at $\sim 7.4 \mathrm{GPa}$ and its evolution upon pressurization. 
from a quasi-layered (2D) to a closely packed (3D) network amorphous. The progress of this transformation, and the pressures over which it occurs, compares well with the IR spectroscopy measurements on stoichiometric a- $\mathrm{As}_{2} \mathrm{Se}_{3}$ performed by Struzhkin et $a l .{ }^{32}$ It is noted that in XRD patterns a very broad peak (as marked by arrow) appears at $\sim 7 \mathrm{GPa}$, which is an additional indication for the change from a quasi-layered 2D to a 3D network. After $7 \mathrm{GPa}$, the peak starts getting more pronounced and gains a reasonable height after $\sim 16 \mathrm{GPa}$. This behavior is consistent with the plot of $\lambda / 2 \sin \theta$ for the FSDP as a function of pressure (Fig. 2), in which three distinct regions are detectable, and highlighted by solid red, green, and blue lines. The slope of the red line (i.e., $-0.004 \mathrm{~nm} / \mathrm{GPa}$ ) indicates that the volume reduces quickly as compared to the reduction of the volume in the pressure regimes indicated by the green (slope, $-0.001 \mathrm{~nm} /$ $\mathrm{GPa}$ ) and blue (slope, $-0.0007 \mathrm{~nm} / \mathrm{GPa}$ ) lines. In the pressure regime indicated by red line (i.e., $\sim 0-7 \mathrm{GPa}$ ), the IRO is quickly destructed due to the enhancement in the interlayer interactions and the structure starts transforming from quasi-layered $2 \mathrm{D}$ to $3 \mathrm{D}$ network. In the green line pressure domain (i.e., from $\sim 7 \mathrm{GPa}$ to $\sim 16 \mathrm{GPa}$ ), quasi-layered $2 \mathrm{D}$ network still persists along with the 3D network, and quasilayered 2D network amorphous is completely transformed into 3D network amorphous at the end of pressure regime (i.e., at $\sim 16 \mathrm{GPa}$ ). In other words, complete disappearance of IRO is confirmed at $\sim 16 \mathrm{GPa}$. After $\sim 16 \mathrm{GPa}$, up to the maximum pressure achieved in the XRD measurements, pressurization causes the gradual densification of the $3 \mathrm{D}$ amorphous as indicated by the blue line.

Figures 3(a) and 3(b) show the high-pressure behavior of a-AsSe during compression and decompression cycles, respectively, observed via Raman spectroscopy. At ambient pressure, the Raman spectrum of a-AsSe consists of a main asymmetric envelope around $220 \mathrm{~cm}^{-1}$, corresponding to the interlayer vibrations of pyramidal $\mathrm{AsSe}_{3}$ units (which are the basic building blocks of the quasi-layered structure in aAsSe). ${ }^{38}$ When the pressure is increased, the intensity of the main envelope decreases and shifts to higher frequencies. The major reduction in the intensity of the main envelope takes place up to $\sim 7.6 \mathrm{GPa}$, and it finally vanishes at

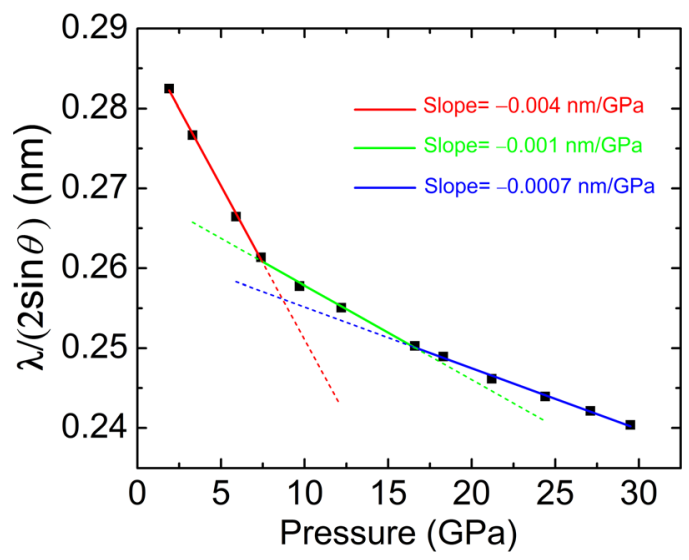

FIG. 2. Data of $\lambda / 2 \sin \theta$ of the FSDP in high-pressure XRD patterns of aAsSe at room temperature as a function of pressure, which is related to the reduced volume of the sample $(\mathrm{V}(\mathrm{P})) /(\mathrm{V}(0))$.
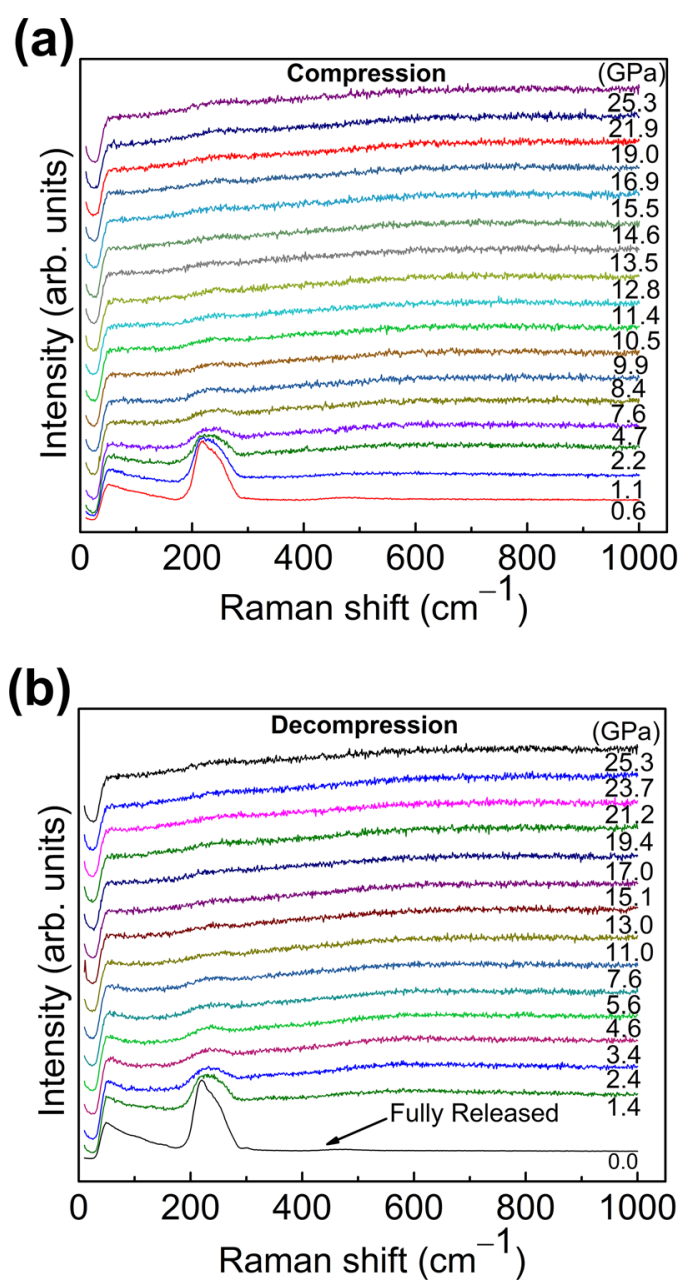

FIG. 3. In-situ high pressure Raman spectroscopy results for a-AsSe at room temperature. (a) Raman spectra at selected pressures during compression up to $25.3 \mathrm{GPa}$. (b) Raman spectra at selected pressures during decompression from $25.3 \mathrm{GPa}$ to $0 \mathrm{GPa}$.

$\sim 14 \mathrm{GPa}$, suggesting the completion of the quasi-layered 2D to $3 \mathrm{D}$ network transformation. As the layers are forced closer, the $\mathrm{AsSe}_{3}$ pyramidal units are distorted (i.e., the AsSe-As bond angle is changed). As a result, the band representing the vibrations of $\mathrm{AsSe}_{3}$ pyramidal units is broadened and reduced in intensity. This behavior is in good agreement with the XRD results in Fig. 1. During decompression, the main envelope of the parent amorphous phase starts to appear back at $\sim 11 \mathrm{GPa}$. With further decrease in pressure, at $\sim 1.4 \mathrm{GPa}$ there is a significant increase in the intensity of the main envelope. Finally, upon complete release of pressure, the main envelope which is the indicative of the parent amorphous phase re-gain its shape. This suggests that the pressure-induced amorphous-to-amorphous network transformation in a-AsSe is reversible upon complete pressure release. Similar reversibility in pressure-induced amorphousto-amorphous transformation has also been observed in other chalcogenide glasses such as $\mathrm{GeSe}_{4}, \mathrm{GeS}_{2}, \mathrm{Ge}_{2.5} \mathrm{As}_{51.25}$ $\mathrm{S}_{46.25}$, and iso-structural $\mathrm{As}_{2} \mathrm{~S}_{3}$ glasses. $18,22,28,30$

The nature of the phase transformation demonstrated in the above mentioned experiments has been analyzed from the variations in local structures point of view by using $a b$ initio molecular dynamic (AIMD) simulations. Fig. 4 shows 

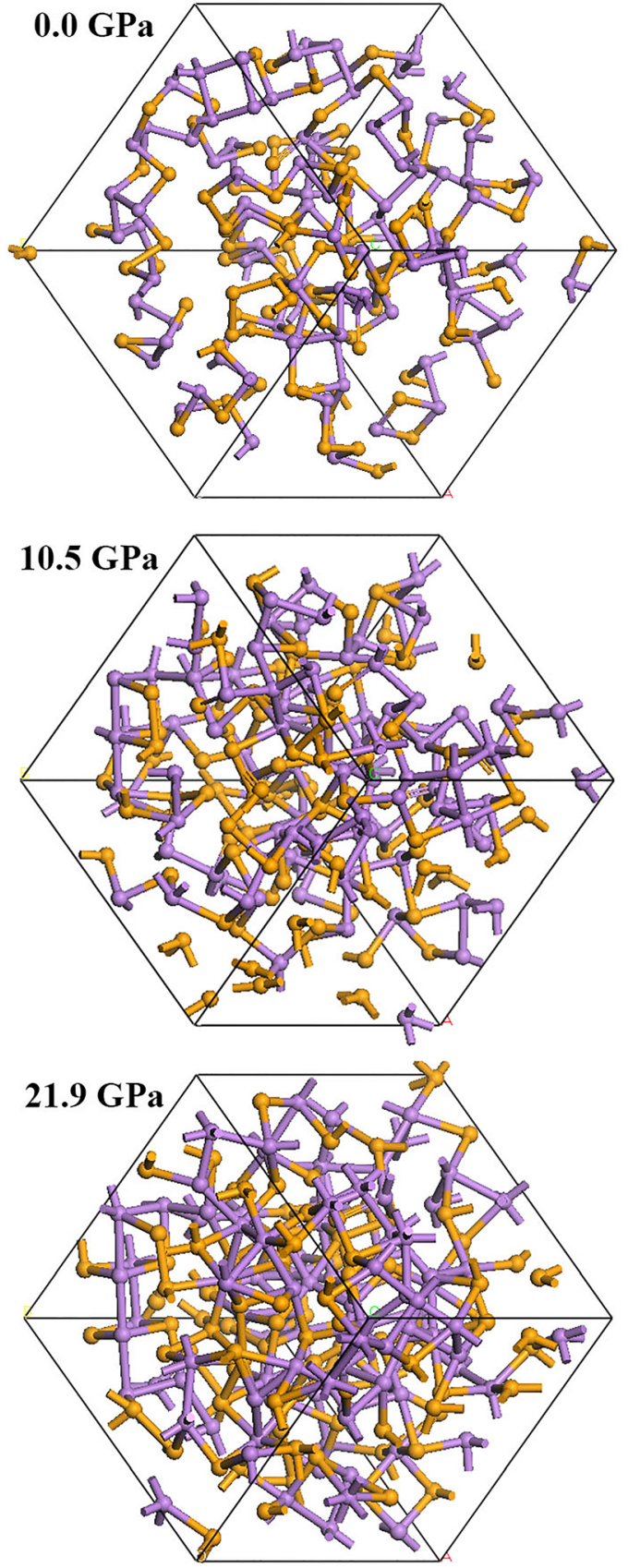

FIG. 4. Optimized atomic configurations obtained from the high pressure ab-initio molecular-dynamics simulations of a-AsSe at various selected pressures. The violet balls represent As atoms and yellow balls represent Se atoms.

a visual representation of quasi-layered (2D) to closely packed (3D) network transformation by showing optimized atomic configurations at three selected pressures. At $0 \mathrm{GPa}$, quasi-layered (2D) structure is obvious, whereas the structure appears more 3D-like in appearance at elevated pressures (e.g., 10.5 GPa and $21.9 \mathrm{GPa}$ ). Fig. 5 shows total and partial As-As, As-Se, and Se-Se pair correlation functions (PCFs) obtained by AIMD simulations. The intensity of the first peak in total PCF decreases with the increase in pressure, while the intensity of the second peak tends to increase with the pressure tuning. The first peak in PCF is found to move towards, higher " $r$ " values, whereas the second peak is found towards lower " $r$ " values (i.e., the first and second

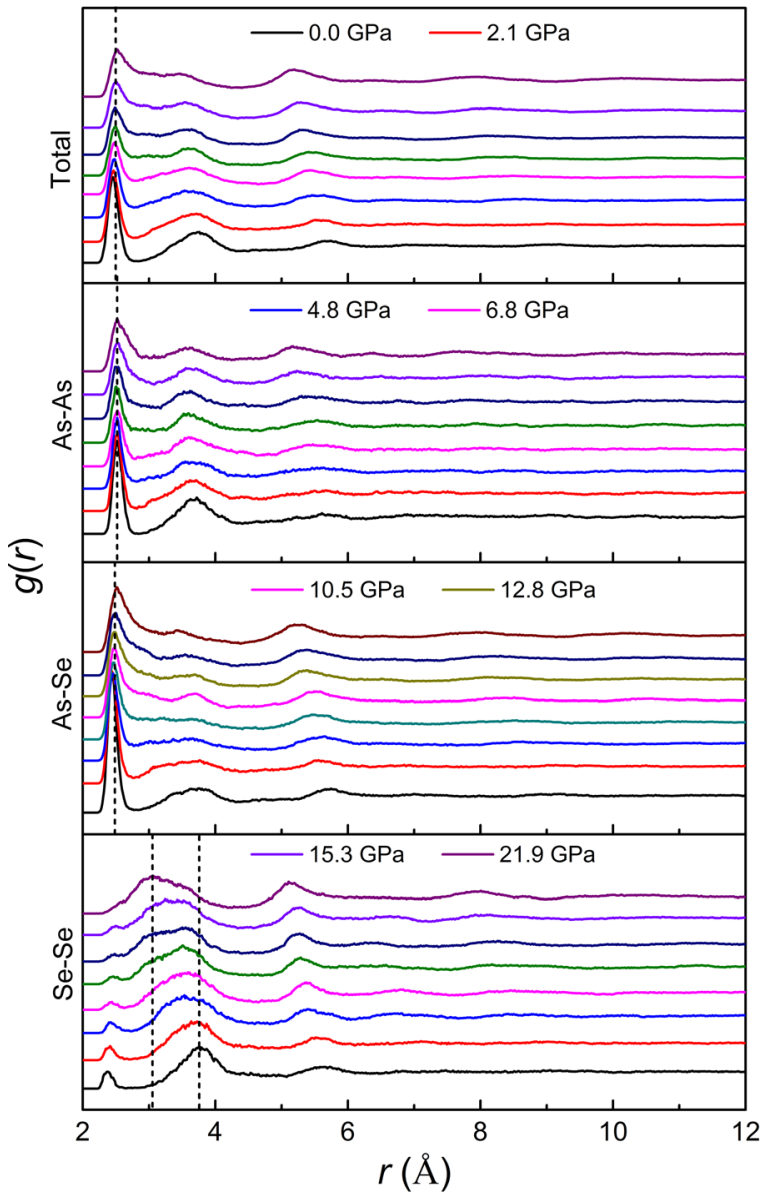

FIG. 5. Total and partial pair correlation functions at various selected pressures as calculated from ab-initio molecular-dynamics simulations of aAsSe during compression up to $21.9 \mathrm{GPa}$. The dashed lines in the total, partial As-As, and partial As-Se pair correlation functions represent the approximate peak position of the first peak in the total, partial As-As, and partial As-Se pair correlation functions, whereas two dashed lines in partial Se-Se pair correlation function represent the approximate initial/final $(3.8 \AA / 3.2 \AA)$ positions of the first most prominent peak in the partial Se-Se pair correlation functions.

peaks are found to merge into each other). This behavior of the first and second peaks under pressure indicates the weakening of the covalent bonding, and hereby the enhancement in the van der Walls forces due to the increased interlayer interaction. A similar behavior of the first and second peaks of $\mathrm{g}(\mathrm{r})$ has been observed in previous experimental studies on glassy $\mathrm{GeSe}_{4}{ }^{22}$ and liquid $\mathrm{GeSe}_{2} .{ }^{10}$ Concerning As-As, As-Se, and Se-Se PCFs, it is found that the Se-Se PCFs are the most affected, while the As-As PCFs are the least affected as a function of pressure. The first and second peaks of Se-Se PCFs are found to merge into each other with increasing pressure (Fig. 5). The second peak in Se-Se PCFs changes its position from an initial position at $\sim 3.8 \AA$ at pressure value of $0.0001 \mathrm{GPa}$ to the final position at $3.2 \AA$ at pressure value of $\sim 22 \mathrm{GPa}$. It means, as the quasi-layered 2D to 3D network transformation proceeds, the Se-Se PCFs are the most affected, pointing to the major player during the transformation.

To investigate the local structure changes associated with the quasi-layered 2D to 3D network transformation, we calculated the total and partial coordination numbers. The 
coordination numbers (CNs) have been calculated by using radial distribution function (RDF) method, in which we integrated $4 \pi r^{2} \rho_{0} g(r)$ to the corresponding peak position of total and partial $\mathrm{g}(\mathrm{r})$ and multiplying it by 2 (i.e., $C . N=2$ $\left.\times \int_{0}^{r_{0}} 4 \pi \mathrm{r}^{2} \rho_{0} \mathrm{~g}(\mathrm{r})\right)$. For the total and partial As-As and As-Se $\mathrm{CNs}$, we integrated it up to the first atomic shell peak positions, whereas for the partial Se-Se CN we integrated it up to the 2 nd atomic shell peak position. This method of calculating the coordination numbers has been successfully applied in the previous studies. ${ }^{39,40}$ Pressure-induced variations in the total, As-Se and Se-Se partial coordination numbers are seemed to follow three trends in three pressure regimes (Fig. 6). The numerical values of pressures that onset these regimes are nearly the same to that of the pressure values that onset the regimes seen in XRD experiment (Fig. 2). The average value of 2 nd shell $\mathrm{Se}-\mathrm{Se}$ coordination number is $\sim 5.1$ at $0 \mathrm{GPa}$ and is increased to $\sim 6.8$ at $\sim 7 \mathrm{GPa}$, while it remains almost unchanged in the pressure range of $\sim 7-15 \mathrm{GPa}$. Further increase in pressure results in the decrease of the 2 nd shell $\mathrm{Se}-\mathrm{Se}$ coordination number from $\sim 6.9$ at $\sim 15 \mathrm{GPa}$ to $\sim 6.3$ at $\sim 22 \mathrm{GPa}$. The average value of the 1 st shell As-Se coordination number decreases from $\sim 3.1$ at $0 \mathrm{GPa}$ to $\sim 2.8$ at $\sim 7 \mathrm{GPa}$, and then it slightly increases from $\sim 2.8$ to $\sim 3$ in the pressure range of $\sim 7-15 \mathrm{GPa}$. With further increase in pressure, the average value of the 1st shell As-Se coordination number is further increased from $\sim 3$ at $\sim 15 \mathrm{GPa}$ to $\sim 4.3$ at $\sim 22 \mathrm{GPa}$. In talking about the average value of the 1 st shell As-As coordination number, it increases gradually from $\sim 1.1$ at $0 \mathrm{GPa}$ to $\sim 1.6$ at $\sim 22 \mathrm{GPa}$. On the other hand, the 1 st shell total average coordination number initially reduces from $\sim 2.2$ at $0 \mathrm{GPa}$ to $\sim 2$ at $\sim 7 \mathrm{GPa}$, increases from $\sim 2$ at $\sim 7 \mathrm{GPa}$ to $\sim 2.5$ at $\sim 15 \mathrm{GPa}$, and then finally increases more from $\sim 2.5$ at $\sim 15 \mathrm{GPa}$ to $\sim 3.3$ at $\sim 22 \mathrm{GPa}$. It can be concluded that as the quasi-layered $2 \mathrm{D}$ to $3 \mathrm{D}$ network transformation proceeds,

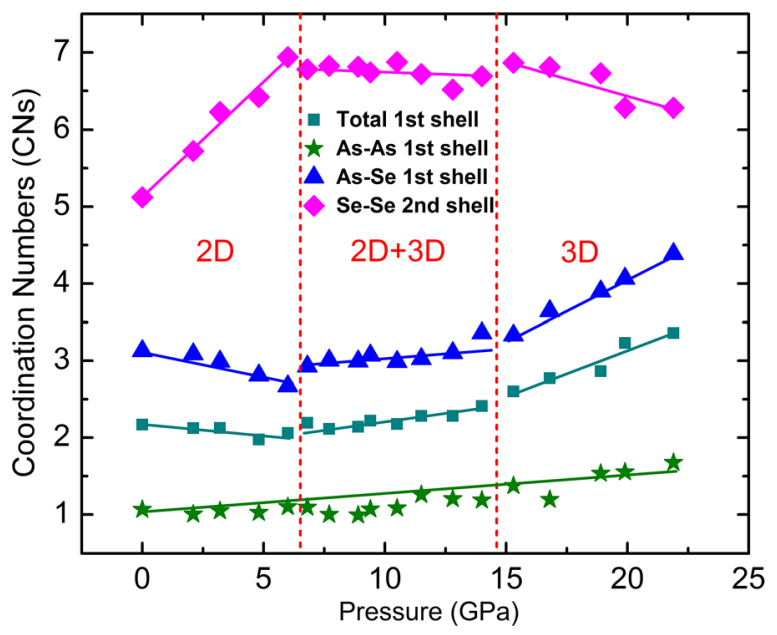

FIG. 6. Pressure dependence of average total and partial coordination numbers as calculated from ab-initio molecular-dynamics simulations of a-AsSe during compression up to $21.9 \mathrm{~Pa}$. The average total and partial As-As, AsSe coordination numbers correspond to the first shell coordination, whereas the partial Se-Se coordination number corresponds to the second shell coordination. The solid lines are guide to the eyes along the various trends in the data. The red color dashed lines separate the transformation process into three pressure regimes. similar to the pair correlation functions (Fig. 5), Se-Se coordination is the most affected, while As-As coordination is the least affected under pressure.

\section{CONCLUSIONS}

In summary, we performed in-situ high pressure XRD, Raman spectroscopic measurements, and ab-initio molecular dynamic simulations on stoichiometric a-AsSe chalcogenide. We observed a gradual densification upon compression up to $\sim 30 \mathrm{GPa}$ along with breakdown of IRO associated with the quasi-layered $2 \mathrm{D}$ to $3 \mathrm{D}$ network transformation at $16 \mathrm{GPa}$. The gradual densification and associated network transformation under pressure can be divided into three pressure regimes: (i) $0-7 \mathrm{GPa}$ pressure regime: in this pressure regime, IRO quickly collapses due to enhancement in interlayer interactions, and quasi-layered (2D) network amorphous starts transforming into 3D network amorphous, but overall structure still remains quasi-layered 2D network amorphous in this regime. (ii) 7-16 GPa pressure regime: in this pressure regime, quasi-layered 2D network amorphous is completely transformed into $3 \mathrm{D}$ network amorphous. (iii) 16-30 GPa pressure regime: in this pressure regime, densification of $3 \mathrm{D}$ network amorphous occurs. In the aforementioned three pressure regimes, volume and coordination numbers of the specimen change with different paces. The amorphous-to-amorphous transformation under compression is found to be reversible upon decompression. Such kind of structural transformation in the intermediate range ordering as a function of pressure is expected to occur in other amorphous As-Se systems as well. Our current work will help the materials scientists and physicist to understand the behavior of network glassy/amorphous materials under pressure. Further studies are needed to explore the behavior of the network glasses and liquids under pressure. These studies should address the pressure-induced structural transformations along with the associated pressure-induced variations in the physical properties (e.g., resistivity) of network glasses and liquids.

\section{ACKNOWLEDGMENTS}

The sample studied here is kindly provided by Dr. B. Bureau. Financial supports from the National Basic Research Program of China (2012CB825700), National Natural Science Foundation of China (Nos. 51371157 and U1432105), and the Fundamental Research Funds for the Central Universities are gratefully acknowledged. The computer resources at National Supercomputer Center in Guangzhou and Tianjin and the calculations performed on TianHe-1 (A) are also gratefully acknowledged. Synchrotron radiation experiments were carried out at the light source PETRAIII at DESY, a member of the Helmholtz Association (HGF).

${ }^{1}$ B. Kalkan, T. G. Edwards, S. Raoux, and S. Sen, J. Chem. Phys. 139, 084507 (2013).

${ }^{2}$ M. Xu, Y. Meng, Y. Q. Cheng, H. W. Sheng, X. D. Han, and E. Ma, J. Appl. Phys. 108, 083519 (2010).

${ }^{3}$ Q. Zeng, H. Sheng, Y. Ding, L. Wang, W. Yang, J.-Z. Jiang, W. L. Mao, and H.-K. Mao, Science 332, 1404 (2011). 
${ }^{4}$ H. Liu, L. Wang, X. Xiao, F. De Carlo, J. Feng, H.-K. Mao, and R. J. Hemley, Proc. Natl. Acad. Sci. 105, 13229 (2008).

5 J. S. Tse and D. D. Klug, Science 255, 1559 (1992).

${ }^{6}$ J. S. Tse, D. D. Klug, J. A. Ripmeester, S. Desgreniers, and K. Lagarec, Nature 369, 724 (1994).

${ }^{7}$ R. J. Hemley, A. P. Jephcoat, H. K. Mao, L. C. Ming, and M. H. Manghnani, Nature 334, 52 (1988).

${ }^{8}$ R. J. Hemley, H. K. Mao, P. M. Bell, and B. O. Mysen, Phys. Rev. Lett. 57, 747 (1986)

${ }^{9}$ C. Meade, R. J. Hemley, and H. K. Mao, Phys. Rev. Lett. 69, 1387 (1992).

${ }^{10}$ W. A. Crichton, M. Mezouar, T. Grande, S. Stolen, and A. Grzechnik, Nature 414, 622 (2001)

${ }^{11}$ T. Matsuoka and K. Shimizu, Nature 458, 186 (2009).

${ }^{12}$ X. Jin, X.-J. Chen, T. Cui, H.-K. Mao, H. Zhang, Q. Zhuang, K. Bao, D. Zhou, B. Liu, Q. Zhou, and Z. He, Proc. Natl. Acad. Sci. 113, 2366 (2016).

${ }^{13}$ V. V. Brazhkin and A. G. Lyapin, J. Phys: Condens. Matter 15, 6059 (2003).

${ }^{14}$ P. F. McMillan, M. Wilson, D. Daisenberger, and D. Machon, Nat. Mater. 4, 680 (2005)

${ }^{15}$ S. K. Deb, M. Wilding, M. Somayazulu, and P. F. McMillan, Nature 414, $528(2001)$.

${ }^{16}$ S. K. Lee, P. J. Eng, H.-K. Mao, Y. Meng, M. Newville, M. Y. Hu, and J. Shu, Nat. Mater. 4, 851 (2005).

${ }^{17}$ Q.-S. Zeng, Y. Ding, W. L. Mao, W. Yang, S. V. Sinogeikin, J. Shu, H.-K. Mao, and J. Z. Jiang, Phys. Rev. Lett. 104, 105702 (2010).

${ }^{18}$ M. Vaccari, G. Garbarino, G. Aquilanti, M. V. Coulet, A. Trapananti, S. Pascarelli, M. Hanfland, E. Stavrou, and C. Raptis, Phys. Rev. B 81, 014205 (2010).

${ }^{19}$ M. Durandurdu and D. A. Drabold, Phys. Rev. B 65, 104208 (2002).

${ }^{20}$ S. M. Antao, C. J. Benmore, B. Li, L. Wang, E. Bychkov, and J. B. Parise, Phys. Rev. Lett. 100, 115501 (2008).
${ }^{21}$ L. B. Skinner, C. J. Benmore, S. Antao, E. Soignard, S. A. Amin, E. Bychkov, E. Rissi, J. B. Parise, and J. L. Yarger, J. Phys. Chem. C 116, 2212 (2012).

${ }^{22}$ B. Kalkan, R. P. Dias, C.-S. Yoo, S. M. Clark, and S. Sen, J. Phys. Chem. C 118, 5110 (2014).

${ }^{23}$ S. R. Elliott, Nature 354, 445 (1991).

${ }^{24}$ S. R. Elliott, J. Non-Cryst. Solid 182, 40 (1995).

${ }^{25}$ C. Massobrio and A. Pasquarello, J. Chem. Phys. 114, 7976 (2001).

${ }^{26}$ M. Fábián, E. Sváb, V. Pamukchieva, A. Szekeres, S. Vogel, and U. Ruett, J. Phys: Conf. Ser. 253, 012053 (2010).

${ }^{27}$ M. Bauchy, M. Micoulaut, M. Boero, and C. Massobrio, Phys. Rev. Lett. 110, 165501 (2013).

${ }^{28}$ M. Vaccari, G. Garbarino, S. N. Yannopoulos, K. S. Andrikopoulos, and S. Pascarelli, J. Chem. Phys. 131, 224502 (2009).

${ }^{29}$ V. V. Brazhkin, Y. Katayama, M. V. Kondrin, A. G. Lyapin, and H. Saitoh, Phys. Rev. B 82, 140202 (2010).

${ }^{30}$ B. Kalkan, C. Sonneville, C. Martinet, B. Champagnon, B. G. Aitken, S. M. Clark, and S. Sen, J. Chem. Phys. 137, 224503 (2012).

${ }^{31} \mathrm{~S}$. Minomura, in Solid State Physics under Pressure, edited by S. Minomura (Terra Scientific, Tokyo, 1985), p. 275.

${ }^{32}$ V. V. Struzhkin et al., Phys. Rev. B 77, 165133 (2008).

${ }^{33}$ A. P. Hammersley, S. O. Svensson, M. Hanfland, A. N. Fitch, and D. Hausermann, High Press. Res. 14, 235 (1996).

${ }^{34}$ G. Kresse and J. Furthmüller, Comput. Mater. Sci. 6, 15 (1996).

${ }^{35}$ P. E. Blöchl, Phys. Rev. B 50, 17953 (1994).

${ }^{36}$ G. Kresse and D. Joubert, Phys. Rev. B 59, 1758 (1999).

${ }^{37}$ M. P. Allen and D. J. Tildesley, Computer Simulation of Liquids (Oxford University Press, 1989).

${ }^{38}$ V. Kovanda, M. Vlček, and H. Jain, J. Non-Cryst. Solid 326-327, 88 (2003).

${ }^{39}$ S. Ohmura and F. Shimojo, Phys. Rev. B 83, 134206 (2011).

${ }^{40} \mathrm{~K}$. Tamura and M. Inui, J. Phys.: Condens. Matter 13, R337 (2001). 